\title{
HARDY-COPSON TYPE INEQUALITIES ON TIME SCALES FOR THE FUNCTIONS OF " $n$ " INDEPENDENT VARIABLES
}

\author{
M. SHAHZAD ASHRAF ${ }^{1, *}$, KHURAM ALI KHAN ${ }^{2}$ AND AMMARA NOSHEEN ${ }^{1}$ \\ ${ }^{1}$ Department of Mathematics, The University of Lahore (Sargodha Campus), Pakistan \\ ${ }^{2}$ Department of Mathematics, University of Sargodha, Sargodha, Pakistan \\ ${ }^{*}$ Corresponding author: shahzadashraf30@icloud.com
}

\begin{abstract}
The paper consists of some extensions in Hardy and Copson type inequalities on time scales. The main results are proved by using induction principle, Rules to find derivatives for composition of two functions, Hölder's inequality and Fubini's theorem in time scales settings. The related results and examples are also investigated in seek of applications.
\end{abstract}

\section{INTRODUCTION}

Advancements in inequalities had started since the end of 19th century, which laid the theocratical foundations of approximation methods. Generally it is accepted that the classic work "Inequalities [7]", which appeared in 1934, gave a systematic discipline to a collection of isolated formulas. This work had played a role of a strong stem in continuous growth to modern field of Inequalities.

Many researchers had paid attention to these inequalities since invention of these inequalities. A large number of papers related with extensions, new results, and generalizations can be seen on the topic $[5,8,10]$. Copson in [6] extend weighted Hardy inequality in the following result:

Received 2018-08-04; accepted 2018-10-24; published 2019-03-01.

2010 Mathematics Subject Classification. 26D15, 39A13, 34N05.

Key words and phrases. time Scales; Hardy-Copson inequality; Keller's chain rule.

(C)2019 Authors retain the copyrights of their papers, and all open access articles are distributed under the terms of the Creative Commons Attribution License. 


$$
\begin{aligned}
& \text { If } \varrho>1, \bar{A}(l)=\sum_{i=1}^{l} \frac{a(i) \omega(i)}{\Omega(i)}, \text { then } \\
& \sum_{l=1}^{\infty} \omega(l)(\bar{A}(l))^{\varrho} \leqslant p^{\varrho} \sum_{l=1}^{\infty} \omega(l) a(l)(\bar{A}(l))^{\varrho-1} \leqslant p^{\varrho} \sum_{l=1}^{\infty} \omega(l) a^{\varrho}(l) .
\end{aligned}
$$

In [12] R. P. Agarwal et al., proved some dynamic Hardy inequalities via time scales calculus. Those inequalities as special cases contain some integral and discrete inequalities due to Hardy and Copson. One of their results is stated as:

"Consider $\mathbb{T}$ is a time scale with $\mathrm{a} \in[0, \infty)_{\mathbb{T}}, \varrho>1, \omega$ and $\mathrm{g}$ be non-negative functions.

Further assume that

$$
\Omega(\varsigma)=\int_{a}^{\varsigma} \omega(r) \Delta r, \quad \Omega(\infty)=\infty, \quad \psi(\varsigma):=\int_{a}^{\varsigma} \omega(r) \mathfrak{g}(r) \Delta r, \quad \forall \varsigma \in[a, \infty)_{\mathbb{T}}
$$

and $\int_{a}^{\infty}\left(\frac{\Omega^{\sigma}(\varsigma)}{\Omega(\varsigma)}\right)^{\varrho(\varrho-1)} \omega(\varsigma) g^{\varrho}(\varsigma) \Delta \varsigma$ exists. Then

$$
\int_{a}^{\infty} \frac{\omega(\varsigma)}{\left(\Omega^{\sigma}(\varsigma)\right)^{\varrho}}\left(\psi^{\sigma}(\varsigma)\right)^{\varrho} \Delta \varsigma \leqslant \frac{\varrho}{\varrho-1} \int_{a}^{\infty} \frac{\omega(\varsigma) \mathfrak{g}(\varsigma)}{\Omega^{\varrho-1}(\varsigma)} \psi^{\varrho-1}(\varsigma) \Delta \varsigma
$$

and

$$
\int_{a}^{\infty} \frac{\omega(\varsigma)}{\left(\Omega^{\sigma}(\varsigma)\right)^{\varrho}}\left(\psi^{\sigma}(\varsigma)\right)^{\varrho} \Delta \varsigma \leqslant\left(\frac{\varrho}{\varrho-1}\right)^{\varrho} \int_{a}^{\infty}\left(\frac{\Omega^{\sigma}(\varsigma)}{\Omega(\varsigma)}\right)^{p(p-1)} \omega(\varsigma) g^{\varrho}(\varsigma) \Delta \varsigma . "
$$

Taking into account the worth of inequalities, we are motivated to extend the results of [12] for functions of several variables.

\section{Preliminaries}

A closed non-empty subset of real numbers is called a time scale. Notation to be used for a time scale is $\mathbb{T} . \mathbb{R}, \mathbb{Z}, \mathbb{N}, \mathbb{N}_{0}$, [a,b] are examples of time scales whereas rationals, irrationals and (a,b) are not closed, therefore not time scales. Few basic concepts related to time scales theory, are as under (see [2-4]):

Definition 2.1. Let $\mathbb{T}$ denotes a time scale. For $\varsigma \in \mathbb{T}$, the forward jump operator $\sigma: \mathbb{T} \rightarrow \mathbb{T}$ is defined as

$$
\sigma(\varsigma):=\inf \{z \in \mathbb{T} ; z>\varsigma\}
$$

and the backward jump operator $\rho: \mathbb{T} \rightarrow \mathbb{T}$ is defined as

$$
\rho(\varsigma):=\sup \{z \in \mathbb{T} ; z<\varsigma\}
$$

The point $\varsigma \in \mathbb{T}$ satisfying $\sigma(\varsigma)>\varsigma$ is right-scattered whereas the point $\varsigma$ satisfying $\rho(\varsigma)<\varsigma$ is leftscattered. Points which are simultaneously left and right scattered are isolated. Also, the point $\varsigma$ is called right-dense if $\varsigma<\sup \mathbb{T}$ and $\sigma(\varsigma)=\varsigma$ and is called left-dense if $\varsigma>\inf \mathbb{T}$ and $\rho(\varsigma)=\varsigma$. The points that are left and right dense simultaneously are dense points. 
Definition 2.2. The Graininess function $\mu: \mathbb{T} \rightarrow[0, \infty)$ is defined by,

$$
\mu(\varsigma):=\sigma(\varsigma)-\varsigma
$$

Definition 2.3. Suppose a function $\mathfrak{g}: \mathbb{T} \rightarrow \mathbb{R}$ is satisfying:

(a) $\mathfrak{g}$ is continuous at all right dense $\varsigma \in \mathbb{T}$,

(b) the left hand limits exist and finite at all left dense $\varsigma \in \mathbb{T}$

then, $\mathfrak{g}$ is called right-dense continuous (rd-continuous) in $\mathbb{T}$. The set denoted by $C_{r d}(\mathbb{T})$ contains all rdcontinuous functions.

Remark 2.1. If we exchange the role of left dense points and right dense points in definition of rd-continuous function, we get left dense continuous functions. If function is continuous with respect to both sided dense points, it is continuous function $\forall t \in \mathbb{T}$.

Definition 2.4. Let $\mathfrak{g}: \mathbb{T} \rightarrow \mathbb{R}$ and $\varsigma \in \mathbb{T}$, if $\mathfrak{g}^{\Delta}(\varsigma)$ exists with the condition that, for $\epsilon>0$, there exists a neighborhood $O$ of $\varsigma$ such that

$$
\left|[\mathfrak{g}(\sigma(\varsigma))-\mathfrak{g}(s)]-\mathfrak{g}^{\Delta}(\varsigma)(\sigma(\varsigma)-s)\right| \leq \epsilon|\sigma(\varsigma)-s|,
$$

for all $s \in O$, then $\mathfrak{g}$ is differentiable at $\varsigma$ and is denoted by $\mathfrak{g}^{\Delta}(\varsigma)$.

Theorem 2.1. Assume delta derivatives of $\mathfrak{g}_{1}, \mathfrak{g}_{2}: \mathbb{T} \rightarrow \mathbb{R}$ exist at $\varsigma \in \mathbb{T}$. Then derivative of the product $\mathfrak{g}_{1} \mathfrak{g}_{2}: \mathbb{T} \rightarrow \mathbb{R}$ exists at $\varsigma \in \mathbb{T}$ and satisfies

$$
\left(\mathfrak{g}_{1} \mathfrak{g}_{2}\right)^{\Delta}(\varsigma)=\mathfrak{g}_{1}^{\Delta}(\varsigma) \mathfrak{g}_{2}(\varsigma)+\mathfrak{g}_{1}^{\sigma}(\varsigma) \mathfrak{g}_{2}^{\Delta}(\varsigma)=\mathfrak{g}_{1}(\varsigma) \mathfrak{g}_{2}^{\Delta}(\varsigma)+\mathfrak{g}_{1}^{\Delta}(\varsigma) \mathfrak{g}_{2}^{\sigma}(\varsigma)
$$

Definition 2.5. Consider $\mathfrak{g}$ is rd-continuous function. Then for $\varsigma_{0} \in \mathbb{T}$, the function $\mathfrak{G}$ defined by

$$
\mathfrak{G}:=\int_{\varsigma_{0}}^{\varsigma} \mathfrak{g}(\varsigma) \Delta \varsigma, \text { for } \varsigma \in \mathbb{T}
$$

is called the antiderivative of $\mathfrak{g}$.

Definition 2.6. If $\alpha \in \mathbb{T}$, $\sup \mathbb{T}=\infty$, and $\mathfrak{g}_{1}$ is $r d$-continuous on the interval $[\alpha, \infty)$, then

$$
\int_{\alpha}^{\infty} \mathfrak{g}_{1}(\varsigma) \Delta \varsigma=\lim _{\beta \rightarrow \infty} \int_{\alpha}^{\beta} \mathfrak{g}_{1}(\varsigma) \Delta \varsigma .
$$

Existence of limit gives convergence of the integral elsewise it diverges.

Theorem 2.2. If $\alpha, \beta, \gamma \in \mathbb{T}, c \in \mathbb{R}$ and $\mathfrak{u}_{1}, \mathfrak{u}_{2} \in C_{r d}$, then

(i) $\int_{\alpha}^{\beta} \mathfrak{u}_{1}^{\sigma}(\varsigma) \mathfrak{u}_{2}^{\Delta}(\varsigma) \Delta \varsigma=\left|\mathfrak{u}_{1} \mathfrak{u}_{2}(\varsigma)\right|_{\alpha}^{\beta}-\int_{\alpha}^{\beta} \mathfrak{u}_{1}^{\Delta}(\varsigma) \mathfrak{u}_{2}(\varsigma) \Delta \varsigma$,

(ii) $\int_{\alpha}^{\beta} \mathfrak{u}_{1}(\varsigma) \mathfrak{u}_{2}^{\Delta}(\varsigma) \Delta \varsigma=\left|\mathfrak{u}_{1} \mathfrak{u}_{2}(\varsigma)\right|_{\alpha}^{\beta}-\int_{\alpha}^{\beta} \mathfrak{u}_{1}^{\Delta}(\varsigma) \mathfrak{u}_{2}^{\sigma}(\varsigma) \Delta \varsigma$.

These are known as integration by parts formulae. 
Theorem 2.3 (Chain rule 1). Let $\mathfrak{u}_{2}: \mathbb{R} \rightarrow \mathbb{R}$ be continuous, $\mathfrak{u}_{2}: \mathbb{T} \rightarrow \mathbb{R}$ is delta differentiable on $\mathbb{T}^{\kappa}$ and suppose that $\mathfrak{u}_{1}: \mathbb{R} \rightarrow \mathbb{R}$ is continuously differentiable. Then there exists $c \in[\varsigma, \sigma(\varsigma)]$ with

$$
\left(\mathfrak{u}_{1} \circ \mathfrak{u}_{2}\right)^{\Delta}(\varsigma)=\mathfrak{u}_{1}^{\prime}\left(\mathfrak{u}_{2}(c)\right) \mathfrak{u}_{2}^{\Delta}(\varsigma)
$$

Theorem 2.4 (Chain rule 2). Assume $\mathfrak{u}_{1}: \mathbb{R} \rightarrow \mathbb{R}$ is continuously differentiable and suppose $\mathfrak{u}_{2}: \mathbb{T} \rightarrow \mathbb{R}$ is delta differentiable. Then $\mathfrak{u}_{1} \circ \mathfrak{u}_{2}: \mathbb{T} \rightarrow \mathbb{R}$ is delta differentiable and

$$
\left(\mathfrak{u}_{1} \circ \mathfrak{u}_{2}\right)^{\Delta}(\varsigma)=\left\{\int_{0}^{1} \mathfrak{u}_{1}^{\prime}\left(\mathfrak{u}_{2}(\varsigma)+h \mu(\varsigma) \mathfrak{g}_{2}^{\Delta}(\varsigma)\right) d h\right\} \mathfrak{u}_{2}^{\Delta}(\varsigma)
$$

holds.

Chain rule 2 is given by C. Pötzsche [11], likewise it also appeared in S. Keller's paper [9]. A simple consequence of Theorem 2.4 is given below:

$$
\left(\mathfrak{u}_{1}^{\omega}(\varsigma)\right)^{\Delta}(\varsigma)=\omega \int_{0}^{1}\left\{h \mathfrak{u}_{1}^{\sigma}+(1-h) \mathfrak{u}_{1}(\varsigma)\right\}^{\omega-1} d h \mathfrak{u}_{1}^{\Delta}(\varsigma) .
$$

Theorem 2.5. (Hölder's inequality) Let $\alpha, \beta \in \mathbb{T}$, for $r d$-continuous functions $\mathfrak{g}_{1}, \mathfrak{g}_{2}:[\alpha, \beta] \rightarrow \mathbb{R}$, we have

$$
\int_{\alpha}^{\beta}\left|\mathfrak{g}_{1}(\varsigma) \mathfrak{g}_{2}(\varsigma)\right| \Delta \varsigma \leq\left[\int_{\alpha}^{\beta}\left|\mathfrak{g}_{1}(\varsigma)\right|^{q} \Delta \varsigma\right]^{\frac{1}{q}}\left[\int_{\alpha}^{\beta}\left|\mathfrak{g}_{2}(\varsigma)\right|^{p} \Delta \varsigma\right]^{\frac{1}{p}}
$$

where $p>1$ and $q=p /(p-1)$.

Next, we present Fubini's Theorem on time scales [1].

Theorem 2.6. Let $\mathbb{T}_{1}, \mathbb{T}_{2}$ be two time scales. Suppose $S: \mathbb{T}_{1} \times \mathbb{T}_{2} \rightarrow \mathbb{R}$ is integrable with respect to both time scales. Define $\Phi\left(y_{2}\right)=\int_{\mathbb{T}_{1}} S\left(y_{1}, y_{2}\right) \Delta y_{1}$ for a.e $y_{2} \in \Lambda$ and $\Psi\left(y_{1}\right)=\int_{\mathbb{T}_{2}} S\left(y_{1}, y_{2}\right) \Delta y_{2}$ for a.e $y_{1} \in \mathbb{T}_{1}$. Then $\Phi$ and $\Psi$ are both differentiable in time scales settings and

$$
\int_{\mathbb{T}_{1}} \Delta y_{1} \int_{\mathbb{T}_{2}} S\left(y_{1}, y_{2}\right) \Delta y_{2}=\int_{\mathbb{T}_{2}} \Delta y_{2} \int_{\mathbb{T}_{1}} S\left(y_{1}, y_{2}\right) \Delta y_{1}
$$

In the sequel, we denote $[a, b)_{\mathbb{T}}=[a, b) \cap \mathbb{T}$, where $\mathbb{T}$ be any time scale. Also partial derivatives for $i \in\{1, \ldots, n\}$ are denoted by $\frac{\partial}{\Delta \varsigma_{i}} \mathfrak{g}\left(\varsigma_{1}, \ldots, \varsigma_{n}\right)=\mathfrak{g}^{\Delta_{i}}\left(\varsigma_{1}, \ldots, \varsigma_{n}\right)$. We also assume that the functions are nonnegative, rd-continuous and the integrals considered are assumed to exist.

\section{Dynamic Hardy \& Copson-Type Inequalities via Time Scales for Functions of $n$ INDEPENDENT VARIABLES}

Theorem 3.1. Let $\mathbb{T}_{1}, \ldots, \mathbb{T}_{n}$ denote time scales. For $p>1$ and $i \in\{1, \ldots, n\}$, consider $a_{i} \in[0, \infty)_{\mathbb{T}_{i}}$ and $g: \mathbb{T}_{1} \times \ldots \times \mathbb{T}_{n} \rightarrow \mathbb{R}^{+}$. Let $\lambda_{i}: \mathbb{T}_{i} \rightarrow \mathbb{R}^{+}$be such that $\Lambda_{i}\left(\varsigma_{i}\right):=\int_{a i}^{\varsigma_{i}} \lambda_{i}\left(s_{i}\right) \Delta s_{i}$ and $\psi\left(\varsigma_{1}, \ldots, \varsigma_{n}\right)=$ 
$\int_{a_{1}}^{\varsigma_{1}} \ldots \int_{a_{n}}^{\varsigma_{n}} \prod_{i=1}^{n} \lambda_{i}\left(s_{i}\right) g\left(s_{1}, \ldots, s_{n}\right) \Delta s_{1} \ldots \Delta s_{n}$.

Assume $\Lambda_{i}(\infty)=0$ and the delta integrals

$$
\int_{a_{1}}^{\infty} \ldots \int_{a_{n}}^{\infty} \prod_{i=1}^{n} \frac{\Lambda_{i}^{\sigma_{i}}\left(\varsigma_{i}\right)}{\left[\Lambda_{i}\left(\varsigma_{i}\right)\right]^{p}} \lambda_{i}\left(\varsigma_{i}\right)\left[g\left(\varsigma_{1}, \ldots, \varsigma_{n}\right)\right]^{p} \Delta \varsigma_{1} \ldots \Delta \varsigma_{n} \text { exist. }
$$

Then

$$
\begin{aligned}
\int_{a_{1}}^{\infty} \ldots \int_{a_{n}}^{\infty} \prod_{i=1}^{n} \frac{\lambda_{i}\left(\varsigma_{i}\right)}{\left[\Lambda_{i}^{\sigma_{i}}\left(\varsigma_{i}\right)\right]^{p}}\left[\psi\left(\sigma_{1}\left(\varsigma_{1}\right), \ldots, \sigma_{n}\left(\varsigma_{n}\right)\right)\right]^{p} \Delta \varsigma_{1} \ldots \Delta \varsigma_{n} \\
\leq\left(\frac{p}{p-1}\right)^{n p} \int_{a_{1}}^{\infty} \ldots \int_{a_{n}}^{\infty} \prod_{i=1}^{n}\left(\frac{\Lambda_{i}^{\sigma_{i}}\left(\varsigma_{i}\right)}{\Lambda_{i}\left(\varsigma_{i}\right)}\right)^{p(p-1)} \lambda_{i}\left(\varsigma_{i}\right) g^{p}\left(\varsigma_{1}, \ldots, \varsigma_{n}\right) \Delta \varsigma_{1} \ldots \Delta \varsigma_{n},
\end{aligned}
$$

where $n$ is any positive integer.

Proof. We prove the result by using Principle of mathematical induction.

For $n=1$, statement is true by [12, Theorem 2.1]. Assume for $1 \leq n \leq k$ (3.2) holds. To prove the result for $n=k+1$, Take left hand side of (3.2) in the following form

$$
\int_{a_{1}}^{\infty} \ldots \int_{a_{k}}^{\infty} \prod_{i=1}^{k} \frac{\lambda_{i}\left(\varsigma_{i}\right)}{\left[\Lambda_{i}^{\sigma_{i}}\left(\varsigma_{i}\right)\right]^{p}}\left\{\int_{a_{k+1}}^{\infty} \frac{\lambda_{k+1}\left(\varsigma_{k+1}\right)}{\left[\Lambda_{k+1}^{\sigma_{k+1}}\left(\varsigma_{k+1}\right)\right]^{p}}\left[\psi\left(\sigma_{1}\left(\varsigma_{1}\right), \ldots, \sigma_{k+1}\left(\varsigma_{k+1}\right)\right)\right]^{p} \Delta \varsigma_{k+1}\right\} \Delta \varsigma_{1} \ldots \Delta \varsigma_{k} .
$$

Consider

$$
I_{k+1}=\int_{a_{k+1}}^{\infty} \frac{\lambda_{k+1}\left(\varsigma_{k+1}\right)}{\left[\Lambda_{k+1}^{\sigma_{k+1}}\left(\varsigma_{k+1}\right)\right]^{p}}\left[\psi\left(\sigma_{1}\left(\varsigma_{1}\right), \ldots, \sigma_{k+1}\left(\varsigma_{k+1}\right)\right)\right]^{p} \Delta \varsigma_{k+1} .
$$

Apply integration by parts (Theorem $2.2(\mathrm{i})$ ) on $I_{k+1}$ w.r.t $\varsigma_{k+1} \in\left[a_{k+1}, \infty\right)$ to get

$$
\begin{aligned}
& I_{k+1}=\left|u_{k+1}\left(\varsigma_{k+1}\right) \psi^{p}\left(\sigma_{1}\left(\varsigma_{1}\right), \ldots, \sigma_{k}\left(\varsigma_{k}\right), \varsigma_{k+1}\right)\right|_{a_{k+1}}^{\infty} \\
& \quad+\int_{a_{k+1}}^{\infty}-u_{k+1}\left(\varsigma_{k+1}\right)\left[\psi^{p}\left(\sigma_{1}\left(\varsigma_{1}\right), \ldots, \sigma_{k}\left(\varsigma_{k}\right), \varsigma_{k+1}\right)\right]^{\Delta_{k+1}} \Delta \varsigma_{k+1},
\end{aligned}
$$

where

$$
\begin{gathered}
-u_{k+1}\left(\varsigma_{k+1}\right)=\int_{\varsigma_{k+1}}^{\infty} \frac{\lambda_{k+1}\left(s_{k+1}\right)}{\left[\Lambda_{k+1}^{\sigma_{k+1}}\left(s_{k+1}\right)\right]^{p}} \Delta s_{k+1} \leq\left(\frac{-1}{p-1}\right) \int_{\varsigma_{k+1}}^{\infty}\left[\Lambda_{k+1}^{1-p}\left(s_{k+1}\right)\right]^{\Delta_{k+1}} \Delta s_{k+1} . \\
\therefore-u_{k+1}\left(\varsigma_{k+1}\right) \leq\left(\frac{1}{1-p}\right)\left(\frac{1}{\Lambda_{k+1}^{p-1}\left(\varsigma_{k+1}\right)}\right) .
\end{gathered}
$$

By chain rule (2.4) and for $d_{k+1} \in\left[\varsigma_{k+1}, \sigma_{k+1}\left(\varsigma_{k+1}\right)\right]$,

$$
\begin{array}{r}
{\left[\psi^{p}\left(\sigma_{1}\left(\varsigma_{1}\right), \ldots, \sigma_{k}\left(\varsigma_{k}\right), \varsigma_{k+1}\right)\right]^{\Delta_{k+1}}} \\
\\
=p \psi^{p-1}\left(\sigma_{1}\left(\varsigma_{1}\right), \ldots, \sigma_{k}\left(\varsigma_{k}\right), d_{k+1}\right) \psi^{\Delta_{k+1}}\left(\sigma_{1}\left(\varsigma_{1}\right), \ldots, \sigma_{k}\left(\varsigma_{k}\right), \varsigma_{k+1}\right) .
\end{array}
$$


Since

$$
\begin{gathered}
\psi^{\Delta_{k+1}}\left(\sigma_{1}\left(\varsigma_{1}\right), \ldots, \sigma_{k}\left(\varsigma_{k}\right), \varsigma_{k+1}\right)= \\
=\int_{a_{1}}^{\sigma_{1}\left(\varsigma_{1}\right)} \ldots \int_{a_{k}}^{\sigma_{k}\left(\varsigma_{k}\right)} \prod_{i=1}^{k} \lambda_{i}\left(s_{i}\right) \times \\
\qquad \frac{\partial}{\Delta \varsigma_{k+1}} \psi\left(\sigma_{1}\left(\varsigma_{1}\right), \ldots, \sigma_{k}\left(\varsigma_{k}\right), \varsigma_{k+1}\right) \\
\quad\left\{\int_{k+1}^{\varsigma_{k+1}} \lambda_{k+1}\left(s_{k+1}\right) g\left(s_{1}, \ldots, s_{k}, s_{k+1}\right) \Delta s_{k+1}\right\} \Delta s_{1} \ldots \Delta s_{k},
\end{gathered}
$$

also

$$
\frac{\partial}{\Delta \varsigma_{k+1}} \int_{a_{k+1}}^{\varsigma_{k+1}} \lambda_{k+1}\left(s_{k+1}\right) g\left(\sigma_{1}\left(s_{1}\right), \ldots, \sigma_{k}\left(s_{k}\right), s_{k+1}\right) \Delta s_{k+1} \geq 0
$$

and $\sigma_{k+1}\left(\varsigma_{k+1}\right) \geq d_{k+1}$. Therefore (3.7) implies

$$
\begin{aligned}
\psi^{\Delta_{k+1}}\left(\sigma_{1}\left(\varsigma_{1}\right), \ldots, \sigma_{k}\left(\varsigma_{k}\right), \varsigma_{k+1}\right) & \\
& =\lambda_{k+1}\left(\varsigma_{k+1}\right) \int_{a_{1}}^{\sigma_{1}\left(\varsigma_{1}\right)} \ldots \int_{a_{k}}^{\sigma_{k}\left(\varsigma_{k}\right)} \prod_{i=1}^{k} \lambda_{i}\left(s_{i}\right) g\left(s_{1}, \ldots, s_{k}, \varsigma_{k+1}\right) \Delta s_{1} \ldots \Delta s_{k} .
\end{aligned}
$$

Thus

$$
\psi^{\Delta_{k+1}}\left(\sigma_{1}\left(\varsigma_{1}\right), \ldots, \sigma_{k}\left(\varsigma_{k}\right), \varsigma_{k+1}\right)=\lambda_{k+1}\left(\varsigma_{k+1}\right) \psi_{k}\left(\sigma_{1}\left(\varsigma_{1}\right), \ldots, \sigma_{k}\left(\varsigma_{k}\right), \varsigma_{k+1}\right)
$$

where

$$
\psi_{k}\left(\sigma_{1}\left(\varsigma_{1}\right), \ldots, \sigma_{k}\left(\varsigma_{k}\right), \varsigma_{k+1}\right)=\int_{a_{1}}^{\sigma_{1}\left(\varsigma_{1}\right)} \ldots \int_{a_{k}}^{\sigma_{k}\left(\varsigma_{k}\right)} \prod_{i=1}^{k} \lambda_{i}\left(s_{i}\right) g\left(s_{1}, \ldots, s_{k}, \varsigma_{k+1}\right) \Delta s_{1} \ldots \Delta s_{k}
$$

Use (3.9) in (3.7) to get,

$$
\begin{aligned}
& {\left[\psi^{p}\left(\sigma_{1}\left(\varsigma_{1}\right), \ldots, \sigma_{k}\left(\varsigma_{k}\right), \varsigma_{k+1}\right)\right]^{\Delta_{k+1}}} \\
& =p \psi^{p-1}\left(\sigma_{1}\left(\varsigma_{1}\right), \ldots, \sigma_{k}\left(\varsigma_{k}\right), d_{k+1}\right) \lambda_{k+1}\left(\varsigma_{k+1}\right) \psi_{k}\left(\sigma_{1}\left(\varsigma_{1}\right), \ldots, \sigma_{k}\left(\varsigma_{k}\right), \varsigma_{k+1}\right) \\
& \quad \leq p \psi^{p-1}\left(\sigma_{1}\left(\varsigma_{1}\right), \ldots, \sigma_{k}\left(\varsigma_{k}\right), \varsigma_{k+1}\right) \lambda_{k+1}\left(\varsigma_{k+1}\right) \psi_{k}\left(\sigma_{1}\left(\varsigma_{1}\right), \ldots, \sigma_{k}\left(\varsigma_{k}\right), \varsigma_{k+1}\right) .
\end{aligned}
$$

$\because \psi\left(\varsigma_{1}, \varsigma_{2}, \ldots, a_{k+1}\right)=0$ and $u_{k+1}(\infty)=0,(3.5)$ becomes

$$
I_{k+1}=\int_{a_{k+1}}^{\infty}-u_{k+1}\left(\varsigma_{k+1}\right)\left[\psi^{p}\left(\sigma_{1}\left(\varsigma_{1}\right), \ldots, \sigma_{k}\left(\varsigma_{k}\right), \varsigma_{k+1}\right)\right]^{\Delta_{k+1}} \Delta \varsigma_{k+1} .
$$

Use (3.6) and (3.10) in (3.11) to get

$$
I_{k+1} \leq\left(\frac{p}{p-1}\right) \int_{a_{k+1}}^{\infty} \frac{\lambda_{k+1}\left(\varsigma_{k+1}\right) \psi_{k}\left(\sigma_{1}\left(\varsigma_{1}\right), \ldots, \sigma_{k}\left(\varsigma_{k}\right), \varsigma_{k+1}\right)}{\Lambda_{k+1}^{p-1}\left(\varsigma_{k+1}\right)}\left[\psi\left(\sigma_{1}\left(\varsigma_{1}\right), \ldots, \sigma_{k+1}\left(\varsigma_{k+1}\right)\right)\right]^{p-1} \Delta \varsigma_{k+1}
$$


Multiply and divide by $\left(\frac{\lambda_{k+1}\left(\varsigma_{k+1}\right)}{\left[\Lambda_{k+1}^{\sigma_{k+1}}\left(\varsigma_{k+1}\right)\right]^{p}}\right)^{\frac{p-1}{p}}$ on right hand side of (3.12) and then apply Hölder's inequality to get

$$
\begin{gathered}
I_{k+1} \leq\left(\frac{p}{p-1}\right)\left(\int _ { a _ { k + 1 } } ^ { \infty } \left(\frac{\lambda_{k+1}\left(\varsigma_{k+1}\right) \Lambda_{k+1}^{1-p}\left(\varsigma_{k+1}\right) \psi_{k}\left(\sigma_{1}\left(\varsigma_{1}\right), \ldots, \sigma_{k}\left(\varsigma_{k}\right), \varsigma_{k+1}\right)}{\left.\left[\left[\Lambda_{k+1}^{\sigma_{k+1}}\left(\varsigma_{k+1}\right)\right]^{-p} \lambda_{k+1} \varsigma_{k+1}\right]^{\frac{p-1}{p}} \Delta \varsigma_{k+1}\right)^{p}}\right.\right. \\
\times\left(\int_{a_{k+1}}^{\infty} \frac{\lambda_{k+1}\left(\varsigma_{k+1}\right)}{\left[\Lambda_{k+1}^{\sigma}\left(\varsigma_{k+1}\right)\right]^{p}}\left[\psi\left(\sigma_{1}\left(\varsigma_{1}\right), \ldots, \sigma_{k+1}\left(\varsigma_{k+1}\right)\right)\right]^{p} \Delta \varsigma_{k+1}\right)^{\frac{p-1}{p}} .
\end{gathered}
$$

Divide both sides by right most term and take power $p$ on both sides. After simplification, we get

$$
I_{k+1}
$$

$$
\leq\left(\frac{p}{p-1}\right)^{p} \int_{a_{k+1}}^{\infty}\left(\frac{\Lambda_{k+1}^{\sigma_{k+1}}\left(\varsigma_{k+1}\right)}{\Lambda_{k+1}\left(\varsigma_{k+1}\right)}\right)^{p(p-1)} \lambda_{k+1}\left(\varsigma_{k+1}\right) \psi_{k}^{p}\left(\sigma_{1}\left(\varsigma_{1}\right), \ldots, \sigma_{k}\left(\varsigma_{k}\right), \varsigma_{k+1}\right) \Delta \varsigma_{k+1} .
$$

Substitute (3.14) in (3.3) to get

$$
\begin{aligned}
& \int_{a_{1}}^{\infty} \ldots \int_{a_{k+1}}^{\infty} \prod_{i=1}^{k+1} \frac{\lambda_{i}\left(\varsigma_{i}\right)}{\left[\Lambda_{i}^{\sigma_{i}}\left(\varsigma_{i}\right)\right]^{p}}\left[\psi\left(\sigma_{1}\left(\varsigma_{1}\right), \ldots, \sigma_{k}\left(\varsigma_{k}\right), \sigma_{k+1}\left(\varsigma_{k+1}\right)\right)\right]^{p} \Delta \varsigma_{1} \ldots \Delta \varsigma_{k} \Delta \varsigma_{k+1} \\
\leq & \left(\frac{p}{p-1}\right)^{p} \int_{a_{1}}^{\infty} \ldots \int_{a_{k}}^{\infty} \prod_{i=1}^{k} \frac{\lambda_{i}\left(\varsigma_{i}\right)}{\left[\Lambda_{i}^{\sigma_{i}}\left(\varsigma_{i}\right)\right]^{p}} \\
& \times\left\{\int_{a_{k+1}}^{\infty}\left(\frac{\Lambda_{k+1}^{\sigma_{k+1}}\left(\varsigma_{k+1}\right)}{\Lambda_{k+1}\left(\varsigma_{k+1}\right)}\right)^{p(p-1)} \lambda_{k+1}\left(\varsigma_{k+1}\right) \psi_{k}^{p}\left(\sigma_{1}\left(\varsigma_{1}\right), \ldots, \sigma_{k}\left(\varsigma_{k}\right), \varsigma_{k+1}\right) \Delta \varsigma_{k+1}\right\} \Delta \varsigma_{1} \ldots \Delta \varsigma_{k} .
\end{aligned}
$$

Use Theorem 2.6 "k times" on right hand side of (3.15) to get

$$
\begin{aligned}
& \int_{a_{k+1}}^{\infty}\left(\frac{\Lambda_{k+1}^{\sigma_{k+1}\left(\varsigma_{k+1}\right)}}{\Lambda_{k+1}\left(\varsigma_{k+1}\right)}\right)^{p(p-1)} \lambda_{k+1}\left(\varsigma_{k+1}\right) \\
& \times\left\{\int_{a_{1}}^{\infty} \ldots \int_{a_{k}}^{\infty} \prod_{i=1}^{k} \frac{\lambda_{i}\left(\varsigma_{i}\right)}{\left[\Lambda_{i}^{\sigma_{i}}\left(\varsigma_{i}\right)\right]^{p}} \psi_{k}^{p}\left(\sigma_{1}\left(\varsigma_{1}\right), \ldots, \sigma_{k}\left(\varsigma_{k}\right), \varsigma_{k+1}\right) \Delta \varsigma_{1} \ldots \Delta \varsigma_{k}\right\} \Delta \varsigma_{k+1} .
\end{aligned}
$$

By using induction hypothesis for $\left.\psi_{k} \sigma_{1}\left(\varsigma_{1}\right), \ldots, \sigma_{k}\left(\varsigma_{k}\right), \varsigma_{k+1}\right)$ (instead for $\psi_{k}\left(\sigma_{1}\left(\varsigma_{1}\right), \ldots, \sigma_{k}\left(\varsigma_{k}\right)\right)$, ) with fix $t_{k+1} \in \mathbb{T}_{k+1}$, in (3.16) and use Fubini's Theorem (Theorem 2.6) "k times" to get

$$
\begin{aligned}
\int_{a_{1}}^{\infty} \ldots \int_{a_{k+1}}^{\infty} \prod_{i=1}^{k+1} \frac{\lambda_{i}\left(\varsigma_{i}\right)}{\left[\Lambda_{i}^{\sigma_{i}}\left(\varsigma_{i}\right)\right]^{p}}\left[\psi\left(\sigma_{1}\left(\varsigma_{1}\right), \ldots, \sigma_{k+1}\left(\varsigma_{k+1}\right)\right)\right]^{p} \Delta \varsigma_{1} \ldots \Delta \varsigma_{k+1} \\
\quad \leq\left(\frac{p}{p-1}\right)^{(k+1) p} \int_{a_{1}}^{\infty} \ldots \int_{a_{k+1}}^{\infty} \prod_{i=1}^{k+1}\left(\frac{\Lambda_{i}^{\sigma_{i}}\left(\varsigma_{i}\right)}{\Lambda_{i}\left(\varsigma_{i}\right)}\right)^{p(p-1)} \lambda_{i}\left(\varsigma_{i}\right) g^{p}\left(\varsigma_{1}, \ldots, \varsigma_{k+1}\right) \Delta \varsigma_{1} \ldots \Delta \varsigma_{k+1} .
\end{aligned}
$$

Thus by principle of mathematical induction, inequality (3.2) holds for all $n \in \mathbb{Z}^{+}$, which completes the proof. 
If we assume

$$
\inf _{\varsigma_{i} \in\left[a_{i}, \infty\right)_{\mathbb{T}_{i}}} \frac{\Lambda_{i}\left(\varsigma_{i}\right)}{\Lambda_{i}^{\sigma_{i}}\left(\varsigma_{i}\right)}=L_{i}>0 \quad \forall i \in\{1, \ldots, n\},
$$

in Theorem 3.1 (in particular in (3.2)), we obtain the following result.

Corollary 3.1. For $p>1$ and $i \in\{1, \ldots, n\}$, consider $\mathbb{T}_{i}$ be time scales, $a_{i} \in[0, \infty)_{\mathbb{T}_{i}}, \lambda_{i}: \mathbb{T}_{i} \rightarrow \mathbb{R}^{+}$and $g: \mathbb{T}_{1} \times \ldots \times \mathbb{T}_{n} \rightarrow \mathbb{R}^{+}$. Let $\Lambda_{i}\left(\varsigma_{i}\right)$ and $\psi\left(\varsigma_{1}, \ldots, \varsigma_{n}\right)$ be defined as in Theorem 3.1 such that (3.17) holds and the delta integrals

$$
\int_{a_{1}}^{\infty} \ldots \int_{a_{n}}^{\infty} \prod_{i=1}^{n} \frac{\Lambda_{i}^{\sigma_{i}}\left(\varsigma_{i}\right)}{\left[\Lambda_{i}\left(\varsigma_{i}\right)\right]^{p}} \lambda_{i}\left(\varsigma_{i}\right)\left[g\left(\varsigma_{1}, \varsigma_{2}, \ldots, \varsigma_{n}\right)\right]^{p} \Delta \varsigma_{1} \Delta \varsigma_{2} \ldots \Delta \varsigma_{n} \quad \text { exist. }
$$

Then (3.2) takes the form

$$
\begin{aligned}
& \int_{a_{1}}^{\infty} \ldots \int_{a_{n}}^{\infty} \prod_{i=1}^{n} \frac{\lambda_{i}\left(\varsigma_{i}\right)}{\left[\Lambda_{i}^{\sigma_{i}}\left(\varsigma_{i}\right)\right]^{p}}[\left.\psi\left(\sigma_{1}\left(\varsigma_{1}\right), \ldots, \sigma_{n}\left(\varsigma_{n}\right)\right)\right]^{p} \Delta \varsigma_{1} \ldots \Delta \varsigma_{n} \\
& \leq\left(\frac{p}{p-1}\right)^{n p} \prod_{i=1}^{n}\left(L_{i}\right)^{p(1-p)} \int_{a_{1}}^{\infty} \ldots \int_{a_{n}}^{\infty} \lambda_{i}\left(\varsigma_{i}\right) g^{p}\left(\varsigma_{1}, \ldots, \varsigma_{n}\right) \Delta \varsigma_{1} \ldots \Delta \varsigma_{n}
\end{aligned}
$$

where " $n$ " is any positive integer.

Remark 3.1. As a special case of Corollary 3.1 when $\mathbb{T}_{i}=\mathbb{R}, \forall i \in\{1, \ldots, n\}$, generalization of integral Hardy inequality for the functions of $\mathrm{n}$ independent variables (note that when $\mathbb{T}_{i}=\mathbb{R}$, we have $\sigma_{i}\left(\varsigma_{i}\right)=\varsigma_{i}$ and $\left.L_{i}=1\right)$ takes the form:

$$
\begin{aligned}
\int_{a_{1}}^{\infty} \ldots \int_{a_{n}}^{\infty} \prod_{i=1}^{n} \frac{\lambda_{i}\left(\varsigma_{i}\right)}{\left[\Lambda_{i}\left(\varsigma_{i}\right)\right]^{p}}\left(\int_{a_{1}}^{\varsigma_{1}} \ldots \int_{a_{n}}^{\varsigma_{n}}\right. & {\left.\left[\prod_{i=1}^{n} \lambda_{i}\left(s_{i}\right) g\left(s_{1}, \ldots, s_{n}\right) d s_{n} \ldots d s_{1}\right]^{p}\right) d \varsigma_{n} \ldots d \varsigma_{1} } \\
& \leq\left(\frac{p}{p-1}\right)^{n p} \int_{a_{1}}^{\infty} \ldots \int_{a_{n}}^{\infty} \prod_{i=1}^{n} \lambda_{i}\left(\varsigma_{i}\right) g^{p}\left(\varsigma_{1}, \ldots, \varsigma_{n}\right) d t_{n} \ldots d \varsigma_{1}
\end{aligned}
$$

As a special case of this inequality when $\lambda_{i}\left(\varsigma_{i}\right)=1, \forall i=\{1, \ldots, n\}$, we have

$$
\begin{array}{r}
\int_{a_{1}}^{\infty} \ldots \int_{a_{n}}^{\infty} \frac{1}{\left[\prod_{i=1}^{n}\left(\varsigma_{i}-a_{i}\right)\right]^{p}}\left(\int_{a_{1}}^{\varsigma_{1}} \ldots \int_{a_{n}}^{\varsigma_{n}}\left[g\left(s_{1}, \ldots, s_{n}\right) d s_{n} \ldots d s_{1}\right]^{p}\right) d \varsigma_{n} \ldots d \varsigma_{1} \\
\leq\left(\frac{p}{p-1}\right)^{n p} \int_{a_{1}}^{\infty} \ldots \int_{a_{n}}^{\infty} g^{p}\left(\varsigma_{1}, \ldots, \varsigma_{n}\right) d \varsigma_{n} \ldots d \varsigma_{1}
\end{array}
$$

where $p>1$.

Remark 3.2. For $p>1$ and $\forall i \in\{1,2, \ldots, n\}$, assume that $\mathbb{T}_{i}=\mathbb{N}$ with $a_{i}=1$ in Corollary 3.1. Furthermore assume that

$$
\sum_{l_{1}=1}^{\infty} \ldots \sum_{l_{n}=1}^{\infty} \lambda_{1}\left(l_{1}\right) \ldots \lambda_{n}\left(l_{n}\right) g^{p}\left(l_{1}, \ldots, l_{n}\right)
$$


is convergent. In this case (3.18) becomes the following discrete Copson type inequality for the functions of " $n$ " independent parameters:

$$
\begin{array}{r}
\sum_{l_{1}=1}^{\infty} \ldots \sum_{l_{n}=1}^{\infty} \frac{\lambda_{1}\left(l_{1}\right) \ldots \lambda_{n}\left(l_{n}\right)}{\left(\sum_{s_{1}=1}^{l_{1}} \lambda_{1}\left(s_{1}\right) \ldots \sum_{s_{n}=1}^{l_{n}} \lambda_{n}\left(s_{n}\right)\right)^{p}}\left(\sum_{s_{1}=1}^{l_{1}} \ldots \sum_{s_{n}=1}^{l_{n}} \lambda_{1}\left(s_{1}\right) \ldots \lambda_{n}\left(s_{n}\right) g\left(s_{1}, \ldots, s_{n}\right)\right)^{p} \\
\leq\left(\frac{p}{p-1}\right)^{k p} \prod_{i=1}^{n}\left(L_{i}\right)^{p(1-p)} \sum_{l_{1}=1}^{\infty} \ldots \sum_{l_{n}=1}^{\infty} \lambda_{1}\left(l_{1}\right) \ldots \lambda_{n}\left(l_{n}\right) g^{p}\left(l_{1}, \ldots, l_{n}\right) .
\end{array}
$$

Theorem 3.2. Let $\mathbb{T}_{1}, \ldots, \mathbb{T}_{n}$ denote time scales. For $p>1$ and $i \in\{1, \ldots, n\}$, consider $a_{i} \in[0, \infty)_{\mathbb{T}_{i}}$ and $g: \mathbb{T}_{1} \times \ldots \times \mathbb{T}_{n} \rightarrow \mathbb{R}^{+}$. Let $\lambda_{i}: \mathbb{T}_{i} \rightarrow \mathbb{R}^{+}, \Lambda_{i}\left(\varsigma_{i}\right):=\int_{a i}^{\varsigma_{i}} \lambda_{i}\left(s_{i}\right) \Delta s_{i}, \Lambda_{i}(\infty)=0$ and the delta integrals

$$
\int_{a_{1}}^{\infty} \ldots \int_{a_{n}}^{\infty} \prod_{i=1}^{n} \lambda_{i}\left(\varsigma_{i}\right)\left[g\left(\varsigma_{1}, \ldots, \varsigma_{n}\right)\right]^{p} \Delta \varsigma_{1} \ldots \Delta \varsigma_{n} \text { exist. }
$$

Assume for any $\varsigma_{i} \in\left[a_{i}, \infty\right)_{\mathbb{T}_{i}}$,

$$
\phi\left(\varsigma_{1}, \ldots, \varsigma_{n}\right):=\int_{\varsigma_{1}}^{\infty} \ldots \int_{\varsigma_{n}}^{\infty} \prod_{i=1}^{n}\left(\frac{\lambda_{i}\left(s_{i}\right)}{\Lambda_{i}^{\sigma_{i}}\left(s_{i}\right)}\right) g\left(s_{1}, \ldots, s_{n}\right) \Delta s_{1} \ldots \Delta s_{n} .
$$

Then

$$
\begin{aligned}
\int_{a_{1}}^{\infty} \ldots \int_{a_{n}}^{\infty} \prod_{i=1}^{n} \lambda_{i}\left(\varsigma_{i}\right)\left[\phi\left(\varsigma_{1}, \ldots, \varsigma_{n}\right)\right]^{p} \Delta \varsigma_{1} \ldots \Delta \varsigma_{n} & \\
& \leq(p)^{n p} \int_{a_{1}}^{\infty} \ldots \int_{a_{n}}^{\infty} \prod_{i=1}^{n} \lambda_{i}\left(\varsigma_{i}\right) g^{p}\left(\varsigma_{1}, \ldots, \varsigma_{n}\right) \Delta \varsigma_{1} \ldots \Delta \varsigma_{n},
\end{aligned}
$$

where $n$ is any positive integer.

Proof. We prove the result by using Principle of mathematical induction.

For $n=1$, statement is true by [12, Theorem 2.5]. Assume for $1 \leq n \leq k$ (3.24) holds. To prove result for $n=k+1$, Take left hand side of (3.24) int the following form

$$
\int_{a_{1}}^{\infty} \ldots \int_{a_{k}}^{\infty} \prod_{i=1}^{k} \lambda_{i}\left(\varsigma_{i}\right)\left\{\int_{a_{k+1}}^{\infty} \lambda_{k+1}\left(\varsigma_{k+1}\right)\left[\phi\left(\varsigma_{1}, \ldots, \varsigma_{k}, \varsigma_{k+1}\right)\right]^{p} \Delta \varsigma_{k+1}\right\} \Delta \varsigma_{1} \ldots \Delta \varsigma_{k} .
$$

Consider

$$
I_{k+1}=\int_{a_{k+1}}^{\infty} \lambda_{k+1}\left(\varsigma_{k+1}\right)\left[\phi\left(\varsigma_{1}, \ldots, \varsigma_{k+1}\right)\right]^{p} \Delta \varsigma_{k+1} .
$$

Apply integration by parts (Theorem $2.2(\mathrm{i})$ ) on $I_{k+1}$ w.r.t $\varsigma_{k+1} \in\left[a_{k+1}, \infty\right)$ to get

$$
I_{k+1}=\left|\phi^{p}\left(\varsigma_{1}, \ldots, \varsigma_{k+1}\right) \Lambda_{k+1}\left(\varsigma_{k+1}\right)\right|_{a_{k+1}}^{\infty}+\int_{a_{k+1}}^{\infty}\left[-\frac{\partial}{\Delta \varsigma_{k+1}}\left[\phi\left(\varsigma_{1}, \ldots, \varsigma_{k+1}\right)\right]^{p} \Lambda_{k+1}^{\sigma_{k+1}}\left(\varsigma_{k+1}\right)\right] \Delta \varsigma_{k+1} .
$$

Since $\phi\left(\varsigma_{1}, \ldots, \varsigma_{k}, \infty\right)=0$ and $\Lambda_{k+1}\left(a_{k+1}\right)=0,(3.27)$ becomes

$$
I_{k+1}=\int_{a_{k+1}}^{\infty}\left[-\frac{\partial}{\Delta \varsigma_{k+1}}\left[\phi\left(\varsigma_{1}, \ldots, \varsigma_{k+1}\right)\right]^{p} \Lambda_{k+1}^{\sigma_{k+1}}\left(\varsigma_{k+1}\right)\right] \Delta \varsigma_{k+1} .
$$


Apply chain rule (2.2) to find upper bound of $-\frac{\partial}{\Delta \varsigma_{k+1}}\left[\phi\left(\varsigma_{1}, \ldots, \varsigma_{k+1}\right)\right]^{p}$, for $d_{k+1} \in$ $\left[\varsigma_{k+1}, \sigma_{k+1}\left(\varsigma_{k+1}\right)\right]$, we get

$$
-\frac{\partial}{\Delta \varsigma_{k+1}}\left[\phi\left(\varsigma_{1}, \ldots, \varsigma_{k+1}\right)\right]^{p}=-p \phi^{p-1}\left(\varsigma_{1}, \ldots, \varsigma_{k}, d_{k+1}\right) \frac{\partial}{\Delta \varsigma_{k+1}} \phi\left(\varsigma_{1}, \ldots, \varsigma_{k}, \varsigma_{k+1}\right) .
$$

Also

$$
\begin{aligned}
& \frac{\partial}{\Delta \varsigma_{k+1}} \phi\left(\varsigma_{1}, \ldots, \varsigma_{k+1}\right)=\frac{\partial}{\Delta \varsigma_{k+1}} \int_{\varsigma_{1}}^{\infty} \ldots \int_{\varsigma_{k+1}}^{\infty} \prod_{i=1}^{k+1}\left(\frac{\lambda_{i}\left(s_{i}\right)}{\Lambda_{i}^{\sigma_{i}}\left(s_{i}\right)}\right) g\left(s_{1}, \ldots, s_{k+1}\right) \Delta s_{1} \ldots \Delta s_{k+1} \\
= & \int_{a_{1}}^{\varsigma_{1}} \ldots \int_{a_{k}}^{\varsigma_{k}} \prod_{i=1}^{k}\left(\frac{\lambda_{i}\left(s_{i}\right)}{\Lambda_{i}^{\sigma_{i}}\left(s_{i}\right)}\right)\left\{\frac{\partial}{\Delta \varsigma_{k+1}} \int_{\varsigma_{k+1}}^{\infty} \frac{\lambda_{k+1}\left(s_{k+1}\right)}{\left.\Lambda_{k+1}^{\sigma_{k+1}\left(s_{k+1}\right)} g\left(s_{1}, \ldots, s_{k+1}\right) \Delta s_{k+1}\right\} \Delta s_{1} \ldots \Delta s_{k}}\right. \\
= & \int_{a_{1}}^{\varsigma_{1}} \ldots \int_{a_{k}}^{\varsigma_{k}} \prod_{i=1}^{k}\left(\frac{\lambda_{i}\left(s_{i}\right)}{\Lambda_{i}^{\sigma_{i}}\left(s_{i}\right)}\right)\left\{\frac{-\lambda_{k+1}\left(\varsigma_{k+1}\right)}{\left.\Lambda_{k+1}^{\sigma_{k+1}\left(\varsigma_{k+1}\right)} g\left(s_{1}, \ldots, s_{k}, \varsigma_{k+1}\right)\right\} \Delta s_{1} \ldots \Delta s_{k}}\right. \\
= & \left(\frac{-\lambda_{k+1}\left(\varsigma_{k+1}\right)}{\Lambda_{k+1}^{\sigma_{k+1}\left(\varsigma_{k+1}\right)}}\right) \int_{a_{1}}^{\varsigma_{1}} \ldots \int_{a_{k}}^{\varsigma_{k}} \prod_{i=1}^{k}\left(\frac{\lambda_{i}\left(s_{i}\right)}{\Lambda_{i}^{\sigma_{i}}\left(s_{i}\right)}\right) g\left(s_{1}, \ldots, s_{k}, \varsigma_{k+1}\right) \Delta s_{1} \ldots \Delta s_{k} .
\end{aligned}
$$

Since $d_{k+1} \geq \varsigma_{k+1}$, use (3.30) in (3.29) to get,

$$
-\frac{\partial}{\Delta \varsigma_{k+1}}\left[\phi\left(\varsigma_{1}, \ldots, \varsigma_{k+1}\right)\right]^{p} \Lambda_{k+1}^{\sigma_{k+1}}\left(\varsigma_{k+1}\right) \leq p \lambda_{k+1}\left(\varsigma_{k+1}\right) \phi^{p-1}\left(\varsigma_{1}, \ldots, \varsigma_{k+1}\right) \phi_{k}\left(\varsigma_{1}, \ldots, \varsigma_{k}\right)
$$

where

$$
\phi_{k}\left(\varsigma_{1}, \ldots, \varsigma_{k}\right)=\int_{a_{1}}^{\varsigma_{1}} \ldots \int_{a_{k}}^{\varsigma_{k}} \prod_{i=1}^{k}\left(\frac{\lambda_{i}\left(s_{i}\right)}{\Lambda_{i}^{\sigma_{i}}\left(s_{i}\right)}\right) g\left(s_{1}, \ldots, s_{k}, \varsigma_{k+1}\right) \Delta s_{1} \ldots \Delta s_{k} .
$$

Then (3.28) becomes,

$$
I_{k+1} \leq p \int_{a_{k+1}}^{\infty} \lambda_{k+1}\left(\varsigma_{k+1}\right) \phi^{p-1}\left(\varsigma_{1}, \ldots, \varsigma_{k+1}\right) \phi_{k}\left(\varsigma_{1}, \ldots, \varsigma_{k}\right)
$$

Multiply and divide by $\left[\lambda_{k+1}\left(\varsigma_{k+1}\right)\right]^{\frac{p-1}{p}}$ on R.H.S of (3.32) then apply Hölder's inequality on R.H.S, we get

$$
\begin{aligned}
I_{k+1} \leq p\left(\int_{a_{k+1}}^{\infty} \lambda_{k+1}\left(\varsigma_{k+1}\right)\left[\phi_{k}\left(\varsigma_{1}, \ldots, \varsigma_{k}, \varsigma_{k+1}\right)\right]^{p} \Delta \varsigma_{k+1}\right)^{\frac{1}{p}} & \\
& \times\left(\int_{a_{k+1}}^{\infty} \lambda_{k+1}\left(\varsigma_{k+1}\right)\left[\phi\left(\varsigma_{1}, \ldots, \varsigma_{k+1}\right)\right]^{p} \Delta \varsigma_{k+1}\right)^{\frac{p-1}{p}} .
\end{aligned}
$$

Divide both sides by right most term then take power $p$ on both sides and after simplification, we get

$$
\int_{a_{k+1}}^{\infty} \lambda_{k+1}\left(\varsigma_{k+1}\right)\left[\phi\left(\varsigma_{1}, \ldots, \varsigma_{k+1}\right)\right]^{p} \Delta \varsigma_{k+1} \leq p^{p} \int_{a_{k+1}}^{\infty} \lambda_{k+1}\left(\varsigma_{k+1}\right)\left[\phi_{k}\left(\varsigma_{1}, \ldots, \varsigma_{k}, \varsigma_{k+1}\right)\right]^{p} \Delta \varsigma_{k+1}
$$


Substitute (3.34) in (3.25), we get

$$
\begin{aligned}
\int_{a_{1}}^{\infty} \ldots \int_{a_{k+1}}^{\infty} & \prod_{i=1}^{k+1} \lambda_{i}\left(\varsigma_{i}\right)\left[\phi\left(\varsigma_{1}, \ldots, \varsigma_{k+1}\right)\right]^{p} \Delta \varsigma_{1} \ldots \Delta \varsigma_{k+1} \\
& \leq p^{p} \int_{a_{1}}^{\infty} \ldots \int_{a_{k}}^{\infty} \prod_{i=1}^{k} \lambda_{i}\left(\varsigma_{i}\right)\left\{\int_{a_{k+1}}^{\infty} \lambda_{k+1}\left(\varsigma_{k+1}\right)\left[\phi_{k}\left(\varsigma_{1}, \ldots, \varsigma_{k+1}\right)\right]^{p} \Delta \varsigma_{k+1}\right\} \Delta \varsigma_{1} \ldots \Delta \varsigma_{k}
\end{aligned}
$$

Use Fubini's Theorem (Theorem 2.6) "k times" on right hand side of (3.35), we get

$$
\begin{aligned}
& \int_{a_{1}}^{\infty} \ldots \int_{a_{k+1}}^{\infty} \prod_{i=1}^{k+1} \lambda_{i}\left(\varsigma_{i}\right)\left[\phi\left(\varsigma_{1}, \ldots, \varsigma_{k+1}\right)\right]^{p} \Delta \varsigma_{1} \ldots \Delta \varsigma_{k+1} \\
& \quad \leq p^{p} \int_{a_{k+1}}^{\infty} \lambda_{k+1}\left(\varsigma_{k+1}\right)\left\{\int_{a_{1}}^{\infty} \ldots \int_{a_{k}}^{\infty} \prod_{i=1}^{k} \lambda_{i}\left(\varsigma_{i}\right) \phi_{k}^{p}\left(\varsigma_{1}, \ldots, \varsigma_{k+1}\right) \Delta \varsigma_{1} \ldots \Delta \varsigma_{k}\right\} \Delta \varsigma_{k+1}
\end{aligned}
$$

By using induction hypothesis for $\phi_{k}\left(\varsigma_{1}, \ldots, \varsigma_{k+1}\right)\left(\right.$ instead $\phi_{k}\left(\varsigma_{1}, \ldots, \varsigma_{k+1}\right)$, with fix $t_{k+1} \in \mathbb{T}_{k+1}$, in $(3.16)$ and again apply Theorem 2.6 "k times" on right hand side to get

$$
\begin{aligned}
\int_{a_{1}}^{\infty} \ldots \int_{a_{k+1}}^{\infty} \prod_{i=1}^{k+1} \lambda_{i}\left(\varsigma_{i}\right)\left[\phi\left(\varsigma_{1}, \ldots, \varsigma_{k+1}\right)\right]^{p} \Delta \varsigma_{1} \ldots \Delta \varsigma_{k+1} \\
\quad \leq p^{(k+1) p} \int_{a_{1}}^{\infty} \ldots \int_{a_{k+1}}^{\infty} \prod_{i=1}^{k+1} \lambda_{i}\left(\varsigma_{i}\right) g^{p}\left(\varsigma_{1}, \ldots, \varsigma_{k+1}\right) \Delta \varsigma_{1} \ldots \Delta \varsigma_{k+1}
\end{aligned}
$$

Thus by principle of mathematical induction (3.24) holds for all positive integers $n$, which completes the proof.

Remark 3.3. As a special case of Theorem 3.2 when $\mathbb{T}_{i}=\mathbb{R}, \forall i \in\{1, \ldots, n\}$, we have following generalization of integral inequality of Copson-type for the functions of " $n$ " independent variables (note that when $\mathbb{T}_{i}=\mathbb{R}$, we have $\phi\left(\sigma\left(\varsigma_{1}\right), \ldots, \sigma\left(\varsigma_{n}\right)\right)=\phi\left(\varsigma_{1}, \ldots, \varsigma_{n}\right), \Lambda_{i}^{\sigma_{i}}\left(\varsigma_{i}\right)=\Lambda_{i}\left(\varsigma_{i}\right)$ and $\left.\mu\left(\varsigma_{i}\right)=0\right)$ and (3.24) takes the form

$$
\begin{aligned}
\int_{a_{1}}^{\infty} \ldots \int_{a_{n}} \prod_{i=1}^{\infty} \lambda_{i}\left(\varsigma_{i}\right)\left(\int_{\varsigma_{1}}^{\infty} \ldots \int_{\varsigma_{n}}^{\infty} \prod_{i=1}^{n} \frac{\lambda_{i}\left(s_{i}\right)}{\Lambda_{i}\left(s_{i}\right)} g\left(s_{1}, \ldots, s_{n}\right) d s_{n} \ldots d s_{1}\right)^{p} d \varsigma_{n} \ldots d \varsigma_{1} \\
\leq p^{n p} \int_{a_{1}}^{\infty} \ldots \int_{a_{n}}^{\infty} \prod_{i=1}^{n} \lambda_{i}\left(\varsigma_{i}\right) g^{p}\left(\varsigma_{1}, \ldots, \varsigma_{n}\right) d \varsigma_{n} \ldots d \varsigma_{1}
\end{aligned}
$$

As a special case of (3.38) when $\lambda_{i}\left(\varsigma_{i}\right)=1, \forall i \in\{1, \ldots, n\}$, we have

$$
\begin{array}{r}
\int_{a_{1}}^{\infty} \ldots \int_{a_{n}}^{\infty}\left(\int_{\varsigma_{1}}^{\infty} \ldots \int_{\varsigma_{n}}^{\infty} \prod_{i=1}^{n} \frac{\lambda_{i}\left(s_{i}\right)}{s_{i}} g\left(s_{1}, \ldots, s_{n}\right) d s_{n} \ldots d s_{1}\right)^{p} d \varsigma_{n} \ldots d \varsigma_{1} \\
\leq p^{n p} \int_{a_{1}}^{\infty} \ldots \int_{a_{n}}^{\infty} g^{p}\left(\varsigma_{1}, \ldots, \varsigma_{n}\right) d \varsigma_{n} \ldots d \varsigma_{1}
\end{array}
$$

where $p>1$. 
Remark 3.4. For $p>1$ and $\forall i \in\{1,2, \ldots, n\}$, assume that $\mathbb{T}_{i}=\mathbb{N}$ with $a_{i}=1$ in Theorem 3.2. Furthermore assume that

$$
\sum_{l_{1}=1}^{\infty} \ldots \sum_{l_{n}=1}^{\infty} \lambda_{1}\left(l_{1}\right) \ldots \lambda_{n}\left(l_{n}\right) g^{p}\left(l_{1}, \ldots l_{n}\right)
$$

is convergent. In this case (3.24) becomes the following discrete Copson type inequality for the functions of " $n$ " independent parameters:

$$
\begin{array}{r}
\sum_{l_{1}=1}^{\infty} \ldots \sum_{l_{n}=1}^{\infty} \lambda_{1}\left(l_{1}\right) \ldots \lambda_{n}\left(l_{n}\right)\left(\sum_{s_{1}=l_{1}}^{\infty} \ldots \sum_{s_{n}=l_{n}}^{\infty} \frac{\lambda_{1}\left(s_{1}\right) \ldots \lambda_{n}\left(s_{n}\right)}{\sum_{s_{1}=1}^{l_{1}} \lambda_{1}\left(s_{1}\right) \ldots \sum_{s_{n}=1}^{l_{n}} \lambda_{n}\left(s_{n}\right)} g\left(s_{1}, \ldots, s_{n}\right)\right)^{p} \\
\leq p^{k p} \sum_{l_{1}=1}^{\infty} \ldots \sum_{l_{n}=1}^{\infty} \lambda_{1}\left(l_{1}\right) \ldots \lambda_{n}\left(l_{n}\right) g^{p}\left(l_{1}, \ldots, l_{n}\right)
\end{array}
$$

Theorem 3.3. Let $\mathbb{T}_{1}, \ldots, \mathbb{T}_{n}$ denote time scales. For $p>1$ and $i \in\{1, \ldots, n\}$, consider $a_{i} \in[0, \infty) \mathbb{T}_{i}$ and $g: \mathbb{T}_{1} \times \ldots \times \mathbb{T}_{n} \rightarrow \mathbb{R}^{+}$. Let $\lambda_{i}: \mathbb{T}_{i} \rightarrow \mathbb{R}^{+}, \Lambda_{i}\left(\varsigma_{i}\right):=\int_{a i}^{\varsigma_{i}} \lambda_{i}\left(s_{i}\right) \Delta s_{i}, \Lambda_{i}(\infty)=0$ and the delta integrals

$$
\int_{a_{1}}^{\infty} \ldots \int_{a_{n}}^{\infty} \prod_{i=1}^{n}\left(\frac{\lambda_{i}\left(\varsigma_{i}\right)}{\Lambda_{i}\left(\varsigma_{i}\right)}\right)\left[g\left(\varsigma_{1}, \ldots, \varsigma_{n}\right)\right]^{p} \Delta \varsigma_{1} \ldots \Delta \varsigma_{n} \text { exist. }
$$

Assume for any $\varsigma_{i} \in\left[a_{i}, \infty\right)_{\mathbb{T}_{i}}$

$$
\phi\left(\varsigma_{1}, \ldots, \varsigma_{n}\right):=\int_{\varsigma_{1}}^{\infty} \ldots \int_{\varsigma_{n}}^{\infty} \prod_{i=1}^{n} \frac{\lambda_{i}\left(s_{i}\right)}{\Lambda_{i}\left(s_{i}\right)} g\left(s_{1}, \ldots, s_{n}\right) \Delta s_{1} \ldots \Delta s_{n} .
$$

Then

$$
\begin{aligned}
& \int_{a_{1}}^{\infty} \ldots \int_{a_{n}}^{\infty} \prod_{i=1}^{n} \lambda_{i}\left(\varsigma_{i}\right)\left[\phi\left(\varsigma_{1}, \ldots, \varsigma_{n}\right)\right]^{p} \Delta \varsigma_{1} \ldots \Delta \varsigma_{n} \\
& \quad \leq(p)^{n p} \int_{a_{1}}^{\infty} \ldots \int_{a_{n}}^{\infty} \prod_{i=1}^{n} \lambda_{i}\left(\varsigma_{i}\right)\left(\frac{\Lambda_{i}^{\sigma_{i}}\left(s_{i}\right)}{\Lambda_{i}\left(s_{i}\right)}\right)^{p} g^{p}\left(\varsigma_{1}, \ldots, \varsigma_{n}\right) \Delta \varsigma_{1} \ldots \Delta \varsigma_{n}
\end{aligned}
$$

where $n$ is any positive integer.

Proof. This result is proved by using Principle of mathematical induction. For $n=1$, statement is true by [12, Theorem 2.8]. Assume for $1 \leq n \leq k$ (3.43) holds. To prove the result for $n=k+1$, Take left hand side of (3.43) in the following form

$$
\int_{a_{1}}^{\infty} \ldots \int_{a_{k}}^{\infty} \prod_{i=1}^{k} \lambda_{i}\left(\varsigma_{i}\right)\left\{\int_{a_{k+1}}^{\infty} \lambda_{k+1}\left(\varsigma_{k+1}\right)\left[\phi\left(\varsigma_{1}, \ldots, \varsigma_{k+1}\right)\right]^{p} \Delta \varsigma_{k+1}\right\} \Delta \varsigma_{1} \ldots \Delta \varsigma_{k} .
$$

Consider

$$
I_{k+1}=\int_{a_{k+1}}^{\infty} \lambda_{k+1}\left(\varsigma_{k+1}\right)\left[\phi\left(\varsigma_{1}, \ldots, \varsigma_{k+1}\right)\right]^{p} \Delta \varsigma_{k+1} .
$$

Use integration by parts Theorem 2.2 (i) with $\phi\left(\varsigma_{1}, \ldots, \varsigma_{k}, \infty\right)=0$ and $\Lambda_{k+1}\left(\varsigma_{k+1}\right)=0$ to get

$$
\int_{a_{k+1}}^{\infty} \lambda_{k+1}\left(\varsigma_{k+1}\right)\left[\phi\left(\varsigma_{1}, \ldots, \varsigma_{k+1}\right)\right]^{p} \Delta \varsigma_{k+1}=\int_{a_{k+1}}^{\infty}-\frac{\partial}{\Delta \varsigma_{k+1}}\left[\phi\left(\varsigma_{1}, \ldots, \varsigma_{k+1}\right)\right]^{p} \Lambda_{k+1}^{\sigma_{k+1}}\left(\varsigma_{k+1}\right) \Delta \varsigma_{k+1}
$$


By chain rule (2.4) for $d_{k+1} \in\left[\varsigma_{k+1}, \varsigma_{k+1}\right]$,

$$
-\frac{\partial}{\Delta \varsigma_{k+1}}\left[\phi\left(\varsigma_{1}, \ldots, \varsigma_{k+1}\right)\right]^{p}=-p \phi^{p-1}\left(\varsigma_{1}, \ldots, \varsigma_{k}, d_{k+1}\right) \frac{\partial}{\Delta \varsigma_{k+1}} \phi\left(\varsigma_{1}, \ldots, \varsigma_{k+1}\right),
$$

and

$$
\begin{aligned}
& \frac{\partial}{\Delta \varsigma_{k+1}} \phi\left(\varsigma_{1}, \ldots, \varsigma_{k+1}\right) \\
& \quad=\int_{\varsigma_{1}}^{\infty} \ldots \int_{\varsigma_{k}}^{\infty} \prod_{i=1}^{k} \frac{\lambda_{i}\left(s_{i}\right)}{\Lambda_{i}\left(s_{i}\right)}\left\{\frac{\partial}{\Delta \varsigma_{k+1}} \int_{a_{k+1}}^{\infty} \frac{\lambda_{k+1}\left(s_{k+1}\right)}{\Lambda_{k+1}\left(s_{k+1}\right)} g\left(s_{1}, \ldots, s_{k+1}\right) \Delta s_{k+1}\right\} \Delta s_{1} \ldots \Delta s_{k} .
\end{aligned}
$$

Also

$$
\frac{\partial}{\Delta \varsigma_{k+1}} \int_{a_{k+1}}^{\infty} \frac{\lambda_{k+1}\left(s_{k+1}\right)}{\Lambda_{k+1}\left(s_{k+1}\right)} g\left(s_{1}, \ldots, s_{k+1}\right) \Delta s_{k+1}=-\frac{\lambda_{k+1}\left(\varsigma_{k+1}\right)}{\Lambda_{k+1}\left(\varsigma_{k+1}\right)} g\left(s_{1}, \ldots, \varsigma_{k+1}\right) \leq 0
$$

and $d_{k+1} \geq \varsigma_{k+1}$. So after simplifications (3.48) implies

$$
\frac{\partial}{\Delta \varsigma_{k+1}} \phi\left(\varsigma_{1}, \ldots, \varsigma_{k+1}\right)=-\frac{\lambda_{k+1}\left(\varsigma_{k+1}\right)}{\Lambda_{k+1}\left(\varsigma_{k+1}\right)} \int_{\varsigma_{1}}^{\infty} \ldots \int_{\varsigma_{k}}^{\infty} \prod_{i=1}^{k} \frac{\lambda_{i}\left(s_{i}\right)}{\Lambda_{i}\left(s_{i}\right)} g\left(s_{1}, \ldots, \varsigma_{k+1}\right) \Delta s_{1} \ldots \Delta s_{k}
$$

Substitute (3.49) in (3.47),

$$
\begin{aligned}
& -\frac{\partial}{\Delta \varsigma_{k+1}}\left[\phi\left(\varsigma_{1}, \ldots, \varsigma_{k+1}\right)\right]^{p} \\
& =p \phi^{p-1}\left(\varsigma_{1}, \ldots, \varsigma_{k}, d_{k+1}\right) \frac{\lambda_{k+1}\left(\varsigma_{k+1}\right)}{\Lambda_{k+1}\left(\varsigma_{k+1}\right)} \int_{\varsigma_{1}}^{\infty} \ldots \int_{\varsigma_{k}}^{\infty} \prod_{i=1}^{k} \frac{\lambda_{i}\left(s_{i}\right)}{\Lambda_{i}\left(s_{i}\right)} g\left(s_{1}, \ldots, \varsigma_{k+1}\right) \Delta s_{1} \ldots \Delta s_{k} \\
& \leq p \phi^{p-1}\left(\varsigma_{1}, \ldots, \varsigma_{k+1}\right) \frac{\lambda_{k+1}\left(\varsigma_{k+1}\right)}{\Lambda_{k+1}\left(\varsigma_{k+1}\right)} \phi_{k}\left(\varsigma_{1}, \ldots, \varsigma_{k+1}\right),
\end{aligned}
$$

where

$$
\phi_{k}\left(\varsigma_{1}, \ldots, \varsigma_{k+1}\right)=\int_{\varsigma_{1}}^{\infty} \ldots \int_{\varsigma_{k}}^{\infty} \prod_{i=1}^{k} \frac{\lambda_{i}\left(s_{i}\right)}{\Lambda_{i}\left(s_{i}\right)} g\left(s_{1}, \ldots, \varsigma_{k+1}\right) \Delta s_{1} \ldots \Delta s_{k} .
$$

Put (3.49) in (3.46),

$$
\begin{aligned}
& \int_{a_{k+1}}^{\infty} \lambda_{k+1}\left(\varsigma_{k+1}\right)\left[\phi\left(\varsigma_{1}, \ldots, \varsigma_{k+1}\right)\right]^{p} \Delta \varsigma_{k+1} \\
& \quad \leq p \int_{a_{k+1}}^{\infty} \lambda_{k+1}\left(\varsigma_{k+1}\right) \frac{\Lambda_{k+1}^{\sigma_{k+1}}\left(\varsigma_{k+1}\right)}{\Lambda_{k+1}\left(\varsigma_{k+1}\right)} \phi^{p-1}\left(\varsigma_{1}, \ldots, \varsigma_{k+1}\right) \phi_{k}\left(\varsigma_{1}, \ldots, \varsigma_{k+1}\right) \Delta \varsigma_{k+1}
\end{aligned}
$$

Multiply and divide by $\left[\lambda_{k+1}\left(\varsigma_{k+1}\right)\right]^{\frac{p-1}{p}}$ on right hand side of (3.51) and apply Hölder's inequality to get

$$
\begin{array}{r}
I_{k+1} \leq p\left(\int_{a_{k+1}}^{\infty} \lambda_{k+1}\left(\varsigma_{k+1}\right)\left(\frac{\Lambda_{k+1}^{\sigma_{k+1}\left(\varsigma_{k+1}\right)}}{\Lambda_{k+1}\left(\varsigma_{k+1}\right)}\right)^{p} \phi_{k}^{p}\left(\varsigma_{1}, \ldots, \varsigma_{k+1}\right) \Delta \varsigma_{k+1}\right)^{\frac{1}{p}} \times \\
\left(\int_{a_{k+1}}^{\infty} \lambda_{k+1}\left(\varsigma_{k+1}\right) \phi^{p}\left(\varsigma_{1}, \ldots, \varsigma_{k+1}\right) \Delta \varsigma_{k+1}\right)^{\frac{p-1}{p}} .
\end{array}
$$


Divide both sides by right most term then take power $p$ on both sides and after simplifying, we get

$$
\begin{aligned}
\int_{a_{k+1}}^{\infty} \lambda_{k+1}\left(\varsigma_{k+1}\right)\left[\phi\left(\varsigma_{1}, \ldots, \varsigma_{k+1}\right)\right]^{p} & \Delta \varsigma_{k+1} \\
& \leq(p)^{p} \int_{a_{k+1}}^{\infty} \lambda_{k+1}\left(\varsigma_{k+1}\right)\left(\frac{\Lambda_{k+1}^{\sigma_{k+1}}\left(\varsigma_{k+1}\right)}{\Lambda_{k+1}\left(\varsigma_{k+1}\right)}\right)^{p} \phi_{k}^{p}\left(\varsigma_{1}, \ldots, \varsigma_{k+1}\right) \Delta \varsigma_{k+1} .
\end{aligned}
$$

Substitute (3.53) in (3.52),

$$
\begin{aligned}
& \int_{a_{1}}^{\infty} \ldots \int_{a_{k+1}}^{\infty} \prod_{i=1}^{k+1} \lambda_{i}\left(\varsigma_{i}\right)\left[\phi\left(\varsigma_{1}, \ldots, \varsigma_{k+1}\right)\right]^{p} \Delta \varsigma_{1} \ldots \Delta \varsigma_{k+1} \\
& \quad \leq(p)^{p} \int_{a_{1}}^{\infty} \ldots \int_{a_{k}}^{\infty} \prod_{i=1}^{k} \lambda_{i}\left(\varsigma_{i}\right)\left\{\int_{a_{k+1}}^{\infty} \lambda_{k+1}\left(\varsigma_{k+1}\right)\left(\frac{\Lambda_{k+1}^{\sigma_{k+1}}\left(\varsigma_{k+1}\right)}{\Lambda_{k+1}\left(\varsigma_{k+1}\right)}\right)^{p} \phi_{k}^{p}\left(\varsigma_{1}, \ldots, \varsigma_{k+1}\right) \Delta \varsigma_{k+1}\right\} \Delta \varsigma_{1} \ldots \Delta \varsigma_{k} .
\end{aligned}
$$

Use Theorem 2.6 "k times" on right hand side of (3.54), to get

$$
=(p)^{p} \int_{a_{k+1}}^{\infty} \lambda_{k+1}\left(\varsigma_{k+1}\right)\left(\frac{\Lambda_{k+1}^{\sigma_{k+1}}\left(\varsigma_{k+1}\right)}{\Lambda_{k+1}\left(\varsigma_{k+1}\right)}\right)^{p}\left\{\int_{a_{1}}^{\infty} \ldots \int_{a_{k}}^{\infty} \prod_{i=1}^{k} \lambda_{i}\left(\varsigma_{i}\right) \phi_{k}^{p}\left(\varsigma_{1}, \ldots, \varsigma_{k+1}\right) \Delta \varsigma_{1} \ldots \Delta \varsigma_{k}\right\} \Delta \varsigma_{k+1}
$$

By using induction hypothesis for $\phi_{k}\left(\varsigma_{1}, \ldots, \varsigma_{k+1}\right)$ (instead $\phi_{k}\left(\varsigma_{1}, \ldots, \varsigma_{k+1}\right)$, with fix $t_{k+1} \in \mathbb{T}_{k+1}$, in (3.55) and again by applying Theorem 2.6 on right hand side, we get

$$
\begin{aligned}
\int_{a_{1}}^{\infty} \ldots \int_{a_{k+1}}^{\infty} \prod_{i=1}^{k+1} \lambda_{i}\left(\varsigma_{i}\right)\left[\phi\left(\varsigma_{1}, \ldots, \varsigma_{k+1}\right)\right]^{p} \Delta \varsigma_{1} \ldots \Delta \varsigma_{k+1} \\
\quad \leq(p)^{(k+1) p} \int_{a_{1}}^{\infty} \ldots \int_{a_{k+1}}^{\infty} \prod_{i=1}^{k+1} \lambda_{i}\left(\varsigma_{i}\right)\left(\frac{\Lambda_{i}^{\sigma_{i}}\left(\varsigma_{i}\right)}{\Lambda_{i}\left(\varsigma_{i}\right)}\right)^{p} g^{p}\left(\varsigma_{1}, \ldots, \varsigma_{k+1}\right) \Delta \varsigma_{1} \ldots \Delta \varsigma_{k+1}
\end{aligned}
$$

Thus by principle of mathematical induction (3.43) holds for all $n \in \mathbb{Z}^{+}$, which completes the proof.

From Theorem 3.3 with condition (3.17), we obtain following result.

Corollary 3.2. For $p>1$ and $i \in\{1, \ldots, n\}$, consider $\mathbb{T}_{i}$ be time scales, $a_{i} \in[0, \infty)_{\mathbb{T}_{i}}, \lambda_{i}: \mathbb{T}_{i} \rightarrow \mathbb{R}^{+}$and $g: \mathbb{T}_{1} \times \ldots \times \mathbb{T}_{n} \rightarrow \mathbb{R}^{+}$. Let $\Lambda_{i}\left(\varsigma_{i}\right)$ and $\psi\left(\varsigma_{1}, \ldots, \varsigma_{n}\right)$ be defined as in Theorem 3.1 such that (3.17) holds and the delta integrals

$$
\int_{a_{1}}^{\infty} \ldots \int_{a_{n}}^{\infty} \prod_{i=1}^{n}\left(\frac{\lambda_{i}\left(\varsigma_{i}\right)}{\Lambda_{i}\left(\varsigma_{i}\right)}\right)\left[g\left(\varsigma_{1}, \ldots, \varsigma_{n}\right)\right]^{p} \Delta \varsigma_{1} \ldots \Delta \varsigma_{n} \quad \text { exists. }
$$

Then

$$
\begin{aligned}
& \int_{a_{1}}^{\infty} \ldots \int_{a_{n}}^{\infty} \prod_{i=1}^{n} \lambda_{i}\left(\varsigma_{i}\right)\left[\phi\left(\varsigma_{1}, \ldots, \varsigma_{n}\right)\right]^{p} \Delta \varsigma_{n} \ldots \Delta \varsigma_{1} \\
& \leq p^{n p} \prod_{i=1}^{n}\left(\frac{1}{L_{i}}\right)^{p} \int_{a_{1}}^{\infty} \ldots \int_{a_{n}}^{\infty} \prod_{i=1}^{n} \lambda_{i}\left(\varsigma_{i}\right) g^{p}\left(\varsigma_{1}, \ldots, \varsigma_{n}\right) \Delta \varsigma_{n} \ldots \Delta \varsigma_{1} .
\end{aligned}
$$


Remark 3.5. As a special case of Corollary 3.2 when $\mathbb{T}_{i}=\mathbb{R}$, integral inequality of Copson-type for the functions of " $n$ " independent variables is

$$
\begin{aligned}
\int_{a_{1}}^{\infty} \ldots \int_{a_{n}}^{\infty} \prod_{i=1}^{n} \lambda_{i}\left(\varsigma_{i}\right)\left(\int_{\varsigma_{1}}^{\infty} \ldots \int_{\varsigma_{n}}^{\infty} \prod_{i=1}^{n} \frac{\lambda_{i}\left(s_{i}\right)}{\Lambda_{i}\left(s_{i}\right)}\right. & \left.g\left(s_{1}, \ldots, s_{n}\right) d s_{n} \ldots d s_{1}\right)^{p} d \varsigma_{n} \ldots d \varsigma_{1} \\
& \leq p^{n p} \int_{a_{1}}^{\infty} \ldots \int_{a_{n}}^{\infty} \prod_{i=1}^{n} \lambda_{i}\left(\varsigma_{i}\right) g^{p}\left(\varsigma_{1}, \ldots, \varsigma_{n}\right) d \varsigma_{n} \ldots d \varsigma_{1},
\end{aligned}
$$

where $p>1$.

Remark 3.6. Assume that $\mathbb{T}_{i}=\mathbb{N}$ in Theorem 3.3, $p>1, a_{i}=1, \forall i \in\{1,2, \ldots, n\}$. Furthermore assume that $\sum_{s_{1}=1}^{\infty} \ldots \sum_{s_{n}=1}^{\infty} \lambda_{1}\left(s_{1}\right) \ldots \lambda_{n}\left(s_{n}\right) \frac{\Lambda_{1}\left(s_{1}+1\right)}{\Lambda_{1}\left(s_{1}\right)} \ldots \frac{\Lambda_{n}\left(s_{n}+1\right)}{\Lambda_{n}\left(s_{n}\right)} g^{p}\left(s_{1}, \ldots, s_{n}\right)$ is convergent. Then (3.43) becomes the following discrete inequality of Copson's type for the functions of " $n$ " independent variables:

$$
\begin{aligned}
\sum_{l_{1}=1}^{\infty} \ldots \sum_{l_{n}=1}^{\infty} \lambda_{1}\left(l_{1}\right) \ldots \lambda_{n}\left(l_{n}\right)\left(\sum_{s_{1}=l_{1}}^{\infty} \ldots \sum_{s_{2}=l_{n}}^{\infty} \frac{\lambda_{1}\left(s_{1}\right) \ldots \lambda_{n}\left(s_{n}\right)}{\Lambda_{1}\left(s_{1}\right) \ldots \Lambda_{n}\left(s_{n}\right)} g\left(s_{1}, \ldots, s_{n}\right)\right)^{p} \\
\quad \leq p^{k p} \sum_{l_{1}=1}^{\infty} \ldots \sum_{l_{n}=1}^{\infty} \frac{\Lambda_{1}\left(l_{1}+1\right) \ldots \Lambda_{n}\left(l_{n}+1\right)}{\Lambda_{1}\left(l_{1}\right) \ldots \Lambda_{n}\left(l_{n}\right)} \lambda_{1}\left(l_{1}\right) \ldots \lambda_{n}\left(l_{n}\right) g^{p}\left(l_{1}, \ldots, l_{n}\right),
\end{aligned}
$$

where $\Lambda_{i}\left(s_{i}\right)=\sum_{a_{i}=1}^{s_{i}-1} \lambda_{i}\left(s_{i}\right), \forall i=1,2, \ldots, n$.

\section{REFERENCES}

[1] M. Bohner, A. Nosheen, J. Peĉarić, A. Younus, Some dynamic Hardy-type inequalities with general kernel, J. Math. Inequal. 8 (2014), 185-199.

[2] M. Bohner and S. G. Georgiev, Multivariable Dynamic calculus on time scales, Springer Intl. Publishing, Switzerland, 2017.

[3] M. Bohner and A. Peterson, Dynamic Equations on time scales: an introduction with Applications, Birkhauser, Boston, MA, 2001.

[4] M. Bohner and A. Peterson, Advances in Dynamic Equations on time scales: an introduction with Applications, Birkhauser, Boston, MA, 2003.

[5] L. Y. Chan, Some extensions of Hardy's inequality, Canad. Math. Bull. 22 (1979), 165-169.

[6] E. T. Copson, Note on series of positive terms, J. London. Math. Soc. 2 (1927), 9-12.

[7] G. H. Hardy, J. E. Littlewood and G. Pólya, Inequalities, 2nd ed., Cambridge University Press, Cambridge, 1952.

[8] S. Kaijser, L. Nikolova, L. E. Persson and A. Wedestig, Hardy-type inequalities via convexity, Math. Inequal. Appl. 8 (2005), 403-417.

[9] S. Keller, Asymotitishces Verhalten invarianter Faserbündel bei Diskretisierung und Mittelwertbildung im Rahmen der Analysis auf Zeitskalen, PhD thesis, Universität Augsburg (1999).

[10] J. Pan, Geometric character of domain and the Harnack inequality of solution of a singular parabolic equation, Math. Slovaca, 62 (2012), 721-734.

[11] C. Pötzsche, Chain rule and invariance principle on measure chains, J. Comput. Appl. Math. 141 (1-2) (2002), 249-254. 
[12] S. H. Saker, D. O'Regan and R. P. Agarwal, Dynamic inequalities of Hardy and Copson type on time scales, Analysis, 34 (4) (2014), 391-402. 